\title{
Technique training of Myofunctional appliance : Activators
}

\section{Hossain MZ1 BDS, PhD}

\begin{abstract}
:
Myofunctional appliance are considered by many authorities orthopedic in nature, influencing the facial skeleton of the growing child in the condylar and suture areas. They also exert an orthodontic effect on the dent-alveolar area. Among these activator is the most well-known myofunctional appliance. The article describes the myofunctional appliance and its technique training, design, steps in preparation of Class II and Class III activator in sequential stages with illustrated case reports, treating class III molar relationship with crowded arch in maxilla and anterior cross bite. The Author acknowledges that the article is summarized from the lectures, handouts during his postgraduate studies in Kyushu University and Hiroshima University, Japan and from his experience from Dhaka Dental College and private practice at Ortho Dental Care. This article is a continuation of the series of technique training in orthodontics especially for the post-gradute trainees as well as for the practitioners who will learn and practice functional appliance specially actvators. Once again, I believe that post-graduate trainee doctors, faculty members, private practitioners and all other concerned will find this article as a guide line during their training as well as in their professional practicing period (Ban J Orthod and Dentofac Orthop, Oct 2011; Vol-2, No. 1, p 34-46)
\end{abstract}

\section{INTODUCTION:}

The Activator itself consists of a loose plastic device fitted on the lingual side of both upper and lower dentition and constructed to a bite which has altered the mandible's functioning position. ${ }^{1}$ Activators are passive appliance to transfer muscle forces from one area to another. Thus they are also considered to be transformator. The uniqueness of functional appliances is their mode of force application. They do not act on the teeth like conventional appliances, using mechanical elements such as springs, elastics, or ligatures, but rather transmit, eliminate or guide natural forces (e.g. muscle activity, growth or tooth eruption). Thus the functional appliance can accelerate the growth, retard the growth or redirect the growth at the level of TMJ and sutures. ${ }^{1}$ This appliance works on two broad principles: ${ }^{2}$

- Force application.

- Force elimination

\section{Force Application}

\section{Force elimination}

Pressure applied on designated area

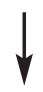

Alteration in form of Involved structure

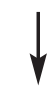

Adaptation in function
This appliance is passive itself but serves as transmitter of forces generated by the oral and facial musculature when used in the oral cavity. Activator is loose fitting removable appliances which redirect the pressures of the facial and masticatory muscle on the tooth and supporting structures to produce improvements in the tooth arrangements and occlusal relations by improving mainly jaws relations. Activator was popularized by the publication of Andresen and Haupl's text on Functional jaw Orthopedics in 1936, although Robin (1902) anticipated the general shape of the appliance 'monobloc' that is used in the Andresen system. ${ }^{3}$ This is referred to as 'activator' as it activates musculature or 'monobloc' or 'andresen appliance' or as 'Norwegian appliance' as it was developed in Norway. Activator is considered the most universal type of functional appliance throughout the world. The upper and lower base plates of this appliance are joined together and bring effect on both the arches, hence it is also called 'monobloc'.

\section{ADVANTAGES:}

1. It eliminates abnormal perioral muscle functions which interfere with normal bone growth.

2. This appliance can be used in mixed dentition without any interference of the eruption of the rest of the permanent teeth.

3. It is a very economic way of rendering service to a large group of patients in growing age, now a days it is being used in non growing individual without which the case would have been very complicated in the adult stage. $4,5,6$

4. These appliance do not have any side effect of mechanotherapy like enamel decalcification, root resorptions etc.

5. It is easier to maintain good oral hygiene.

6. The interval between appointments is relatively long.

7. The appointments are usually less, because it needs minimal adjustments.

8. It is acceptable to many patients because it can be used at night time.

\footnotetext{
${ }^{1}$ Professor and Head, Dept. of Orthodontics and Dentofacial Orthopaedics, Dhaka Dental College and Hospital, Mirpur-14, Dhaka 1206
} 


\section{DISADVANTAGES/ LIMITATIONS}

1. It is mainly used to correct basal bone /arch relationship and to some extent dental alignment. Final detailing of the occlusion can not be given.

2. It has limited used to manage adult patients where the active growth has been ceased because its main function is to modify growth.

3. It is followed by Fixed orthodontic appliance for better occlusal interdigitation because activator cannot produce proper detailing and finishing of occlusions.

4. Success of treatment depends upon the cooperation of the patients because it can be removed by the patients whenever he/she wishes.

5. Severe crowding patients are not indicated, because individual tooth movements are difficult to accomplish. The activator is therefore not recommended for the treatment of malocclusion with crowding and rarely recommended for malocclusion requiring extractions.

6. Mouth breathers have restricted use of this appliance.

7. It is not used in high angle cases.

8. The activator cannot be used in children with abnormal perioral musculature.

\section{INDICATIONS OF ACTIVATOR THERAPY:2,4}

Activator is best suited for the achievement of gross changes in sagittal and vertical dimensions in the mixed and early permanent dentition. The mild to moderate class II div. 1 malocclusion with a deep bite and a horizontal growth direction responds best to treat with an activator. The activator can be used for the partial or total correction of-

1. Class II Div. 1 Malocclusion due to mandibular deficiency.

2. Class II Div. 2 Malocclusion for bite opening.

3. Class III Malocclusion

4. Open bite malocclusion: The dento alveolar anterior and posterior open bite problems are usually attributable to abnormal tongue posture and function and usually respond successfully to activator intervention in mixed dentition.

5. Deep bite cases.

6. Children with lack of vertical development in lower facial height.

7. Midline shifted (skeletal) case

\section{CONTRAINDICATIONS OF ACTIVATOR THERAPY 2,4}

1. Severe crowding (because individual tooth movements are difficult to accomplish. The activator is therefore not recommended for the treatment of malocclusion with crowding and rarely recommended for malocclusion requiring extractions.)

2. Class II Div. 1 malocclusion due to maxillary prognathism.

3. Class III malocclusion when mandible cannot be repositioned backward during functional evaluation or by manually guided.

4. High angle case.

5. Abnormal perioral musculature

6. Non cooperative patients.
Other Special cases considered for activator therapy:

1. Activator can be used successfully in aged patients (nongrowing individuals) if the functional or manual guided position of mandible is comfortable or tolerable for the patients ${ }^{4,5,6}$

2. Deep over bite caused by supraocclusion of the incisors (functional psedu overbite) is not indicated to treat with activator.

3. A skeletal open bite problems, there is a genetically determined vertical growth pattern, which is often associated with marked antegonial notching. Activator is not indicated in such type of cases. Fixed appliance, often with tooth sacrifies, offer a more correctine approach. In extreme cases, ortognathic surgery is the only viable alternative after completion of growth. In early mixed dentition, a partial improvement may be achieved by elimination of some of the dysfunction.

Clinical and laboratory steps in fabrication and treatment with Activator:

1. Proper diagnosis of the case by assessing followings:

a. Model analysis

b. Functional analysis

c. Cephalometric analysis

d. Hand wrist X-rays

e. Cervical vertaeba analysis for growth ${ }^{7}$

2. Visual treatment object (V.T.O)

3. Working Bite registration

a. Class II activator in patient's mouth

b. Class III activator in patient's mouth

4. Recheck the registered bite in plaster model

5. Wire frame work

6. Articulation of the registered bite

7. Fabrication of the appliance

8. Trimming and polishing of the appliance

9. Insertion of the appliance in the oral cavity

10. Instruction for the appliance

11. Selective trimming and adding of the resin as an adjustment of the appliance

\section{Proper diagnosis}

a. Model analysis 8

Following points should be considered:

i. Molar relationship

ii. Mid-line shifting

iii. The symmetry of the dental arches, for example segmental open bite may be corrected with the activator. ${ }^{4}$

iv. Curve of Spee (it can or should be leveled with activator. if the curve of Spee is deep and bicuspid already erupted, the leveling is beyond the capabilities of the activator.)

v. Crowding and any other discrepancies. 
b. Functional analysis. $^{8}$

i. Rest position of the mandible: Vertical opening and antero-posterior repositioning of the mandible during construction bite depends on this.

ii. Mandibular path of closure: any transverse or sagittal deviations are recorded

iii. Premature contact, occlusal interference, and resultant mandibular displacement are checked. Some of the dysfunction can be eliminated with the activator.

iv. TMJ : a functional abnormal cases may need some modification of activator.

v. Interocclusal clearance or Free way space.

vi. Respiration : with allergies or disturbed nasal respiration, the patient cannot wear a bulky appliance ( eliminate cause first or open activator may be used in this case). Adenoid problem should be recorded, patient will not be able to tolerate the activator with adenoid problem.

c. Cephalometric analysis will help in determining the followings 9

i. Direction of growth: average, horizontal or vertical

ii. Differentiation between position and size of the jaws

iii. Morphological peculiarities particularly of mandible.

iv. Axial inclination and position of the maxillary and mandibular incisors.

Cephlometics enable the localization of the apparent anomaly and provides a differentiation between skeletal and dentoalveolar malocclusion which is very much important for treating with activator therapy. For treatment with activator the various Cephalometric measurement can be divided into three groups:

\section{Facial skeleton analysis}

a. The sadle angle: The sadle angle is formed by joining the NS line and ArS line. Its normal value is $123 \pm 5^{\circ}$ The large sadle angle usually means that there is a posterior condylar position and a mandible that is posteriorly positioned with respect to the cranial base and maxilla, is very difficult to influence with activator therapy (functional appliance)

b. Articular angle : lies between the upper and lower parts of the posterior contours of the facial skeleton. Normal value $143 \pm 6^{\circ}$ Increased Ar angle means retrognathic mandible, Decreased means prognathic mandible. The angle can be influenced during activator therapy.

c. The gonial angle: acute or small Go anlge means horizontal growth pattern and favorable to activator therapy. In case of large angle (verticle pattern), activator treatment is contraindicated.

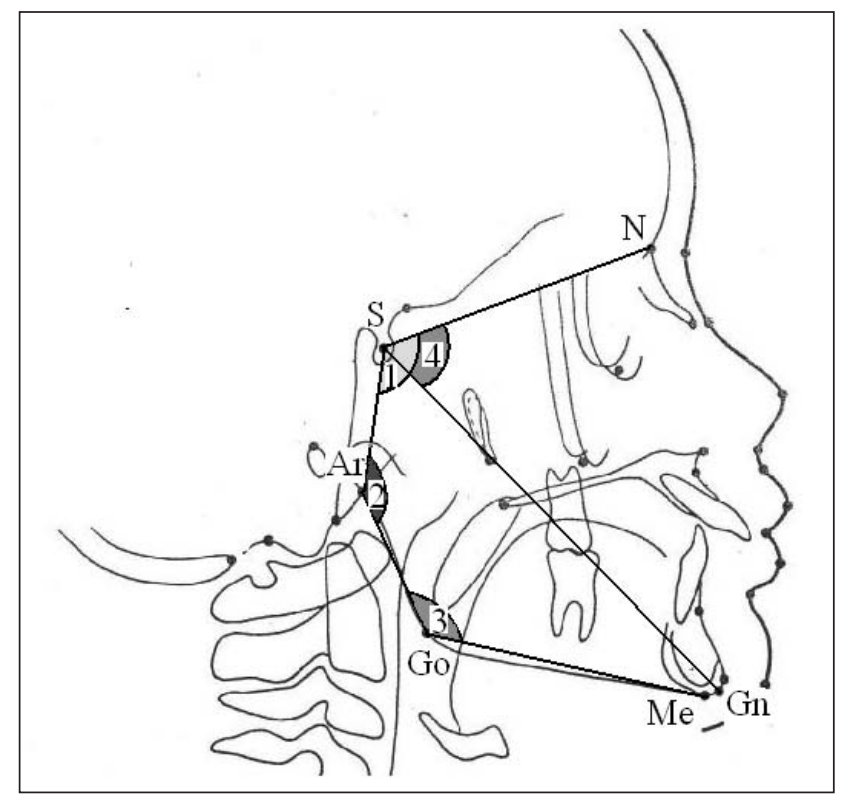

Fig. 1: Sadle angle, formed by joining the NS line and ArS line(1), Articular angle formed by joining points S, Ar and Go (2), Gonial angle fomed by joning the tangentposterior border of the ramus and Go Me line (3) and $\mathrm{Y}$ axix (joining the points $\mathrm{N}, \mathrm{S}$ and $\mathrm{Gn}$ ) : normal value is 85 degree, large angle indicates vertical growth pattern and small angle indicates horizontal growth pattern(4).

d. The posterior facial height(S-Go) and anterior facial height(N-M) ratio: posterior facial height X 100/ anterior facial height

Less than $62 \%$-Vertical growth

More than $65 \%$-Horizontal growth.

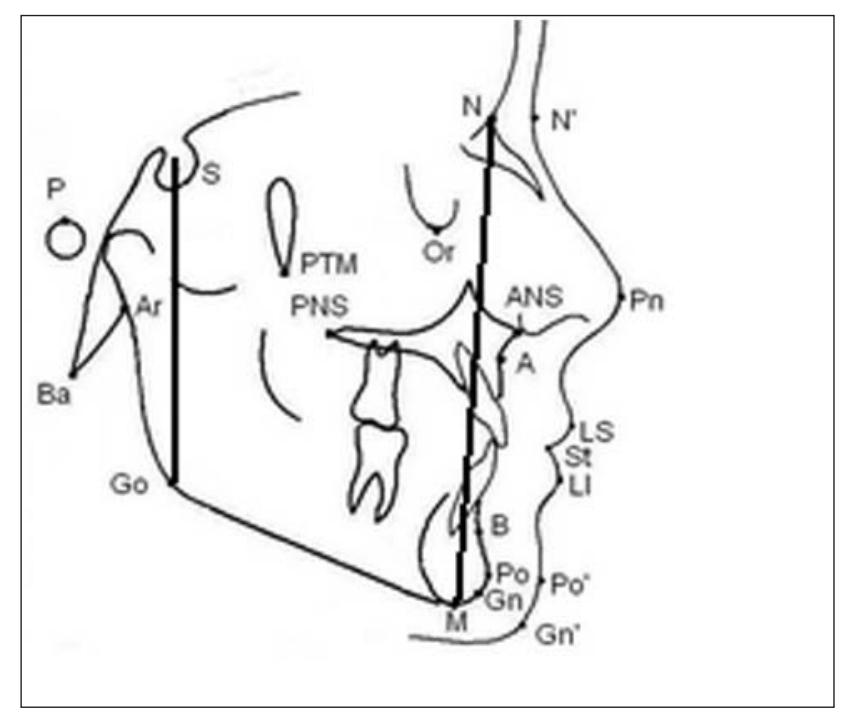

Fig. 2: Anterior facial height (nasion and menton) and Posterior facial height (distance between $\mathrm{S}$ and Go) 


\section{Analysis of the jaw bases}

a. SNA angle expresses the sagittal (antero-posteror) relationship of the anterior limit of the maxillary apical base (Point A) compared to the anterior cranial base. This angle not only inform the antero-posterior relation of maxilla but also inform the growth pattern. SNA angle is large in a prognathic maxilla and the use of class II activator is contraindicated. SNA angle is decreased in retrognathic Maxilla. A moderate decreased of this angle is possible using conventional Class II activator therapy and a larger decreased is indicated for using special activator construction. Normal value of SNA angle is $82 \pm 2^{\circ}$

b. SNB angle is large in prognathic mandible and small in retrognathic mandible. This angle only inform the antero-posterior position of mandible and not morphology or growth direction of mandible. A posteriorly located mandible can be large or small. Normal value $78 \pm 2^{\circ}$

c. Basal plane angle (maxillo-mandibular plane angle) normal value is $25^{\circ}$ It is smaller in horizontal growth pattern and larger in vertical growth pattern.

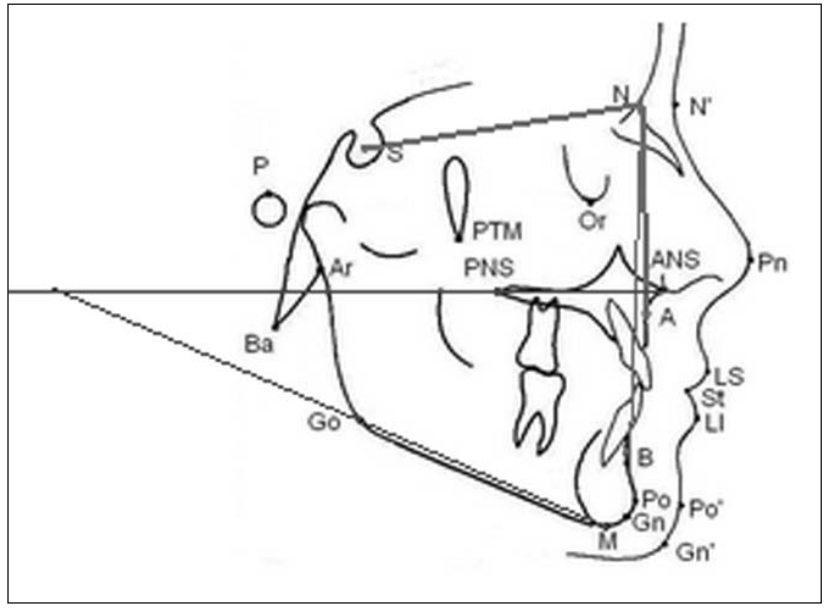

Fig 3: SNA angle (joining points $\mathrm{S}, \mathrm{N}$ and $\mathrm{A}$ ), SNB angle (joining points $\mathrm{S}, \mathrm{N}$ and $\mathrm{B}$ ), and Basal angle Joining palatal plane and base of the mandible

d. Inclination angle is formed by the Pn line (a perpendicular from soft tissue N) and the Palatal plane. Normal value 85 degree. A large angle indicates upward and forward inclination and small angle indicates a downward and backward tipping of maxillary base. Functional appliance can alter the inclination of maxillary base.

Linear measurement of the jaw bases.

S-N : mandibular base $=20: 21$

Ascending ramus : mandibular base $=5: 7$

Maxillary base : mandibular base $=2: 3$
If the mandible is retrognathic, the question arises as to whether the mandible is relatively small or large.This is important when considering the etiology and functional therapy for each patient. The length of maxillary base, mandibular base and ascending ramus are measured relative to the length of the S-N.

Extent of the mandibular base : distance from gonion to pogonion.

Ascending ramus : Gonion to Condylion. It is short in vertical growth pattern.

Maxillary base : Distance between the PNS and point A, projected perpendicular to palatal plane

length of S-N (anterior cranial base length): Distance between the centre of the superior entrance to sella turcica and nasion.

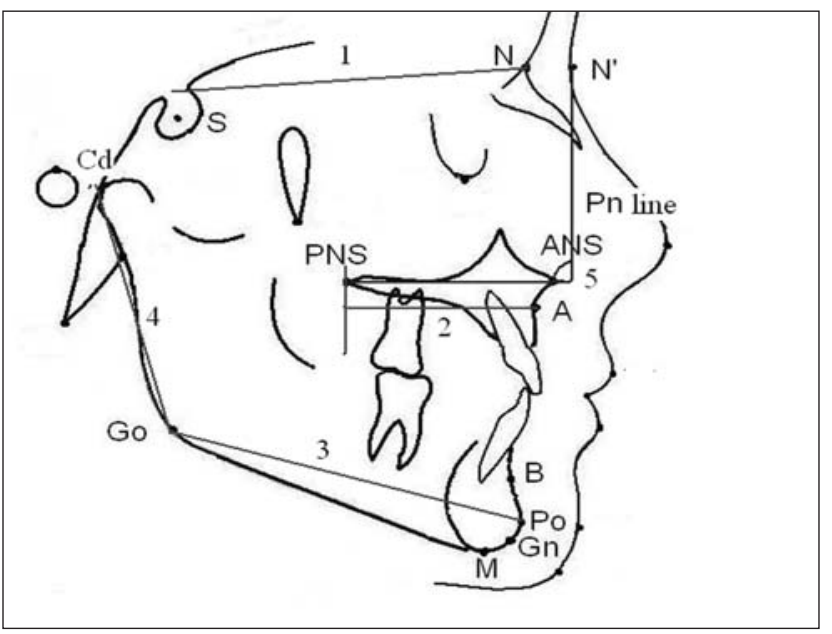

Fig 4: Anterior cranial base length (1), Maxillary base length(2), Mandibular Base length (3), Ascending Ramus (4) and Inclination angle (5)

Morphological peculiarities of mandible:

In orthognathic type of face :

- Fully developed ramus and body of the mandible

- The width of ascending ramus is equal to the height of the body of the mandible

- Condyle and coronoid processes are almost on the same plane

- Symphysis is well developed

In prognathic type of face :

- Body of the mandible is well developed and wide in molar region

- Ramus wide and long

- Gonial angle is small

- Symphysis wider in the sagittal plane

- Mandible grows horizontally. Even if there is average or vertical growth direction in the mixed dentition, a shift of the mandible to a horizontal growth can be expected in the following years. 
In retrognathic type of face :

- Body of the mandible is narrow prticularly in the molar region.

- Ramus is narrow and short

- Coronoid process is shorter than the condylar process

- Symphysis is narrow and long.

- A shift of the growth pattern in the opposite direction is less likely be expected.

\section{Dentoalveolar analysis :}

- Axial inclination of the incisors:

i. The upper incisors: I SN angle is formed by joining the long axix of the maxillary incisors and SN plane. The posterior angle is measured. Normal value $102^{\circ}$ Large angle indicates labial crown tipping.

ii. The lower incisors: I MP angle is formed by joining the long axix of the lower incisors and mandibuylar plane. The posterior angle is measured. Normal value is $90 \pm 3^{\circ}$ Large angle indicates labial crown tipping.

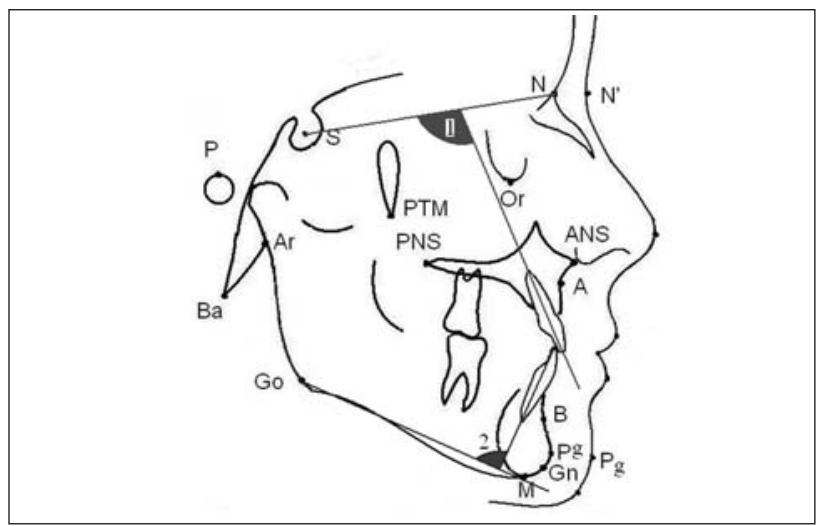

Fig 5: upper incisor inclination (1) and Lower incisor Inclination (2)]

- Position of the Incisors: the most common method is to measure the distance of the incisal edges to the N-Pg line (facial plane). The average position of the upper incisors is 2 $\mathrm{mm}$ to $4 \mathrm{~mm}$ anterior to the Facial plane and lower incisors varies from $2 \mathrm{~mm}$ posterior to $2 \mathrm{~mm}$ anterior to the facial plane.

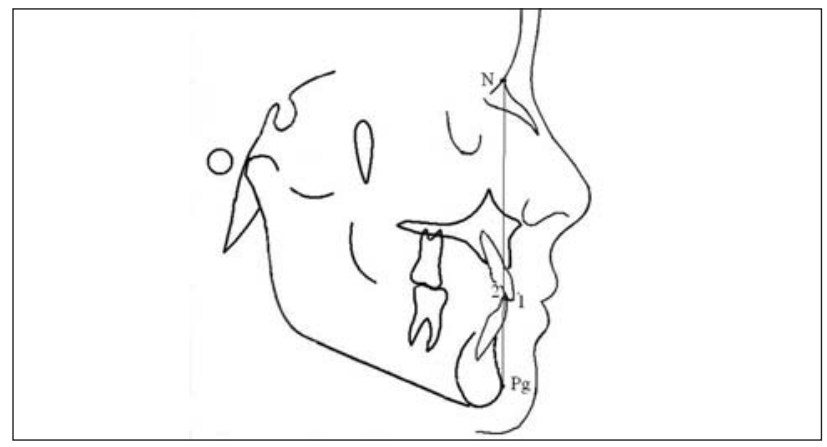

Fig 6: Upper incisor position (1), Lower incisor position (2)
One of the most important tasks of cephalometrics is to objectively assess the changes included by therapy, combined with growth and development, as treatment progress. This should be done periodically.

d. Hand wrist X rays: Should be assessed for the growth status of the patient.The hand - wrist region made up of a. distal ends of radius and ulna, b. carpals, c. metacrapals and d. phalanges. These bones show a predictable or scheduled pattern of appearance, ossification and union from birth to maturity. Thus hand wrist $\mathrm{x}$-ray is important to determined the skeletal maturation status of individuals

Orthodontic treatment for malocclusion moves faster during growth spurts. In general, children have a pattern of fast growth, followed by slow growth in late childhood, and then another growth spurt in the teen years. Because children start this pattern at different ages, chronological age (real age) doesn't indicate a child's stage of development very well. Some children just develop faster or slower than others.

The hand-wrist radiograph, or X-ray image of the wrist bones, can help pinpoint a child's skeletal age. Wrist bones develop to adult size in a clear pattern. This has allowed experts to make a picture atlas of wrist bones in various development stages. Orthodontists can compare a hand-wrist radiograph with the atlas and find out a child's skeletal age. With this data, an orthodontist predicts the next growth spurt and makes a treatment plan that takes advantage of the timing.

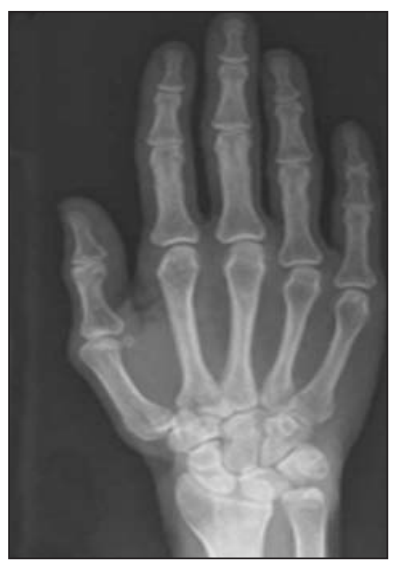

Fig 7:Child's Wrist and Hand, showing the clear lines at the end (epiphysis) of the long bones. These areas, which are made of cartilage, are the epiphyseal plates, where growth occurs.

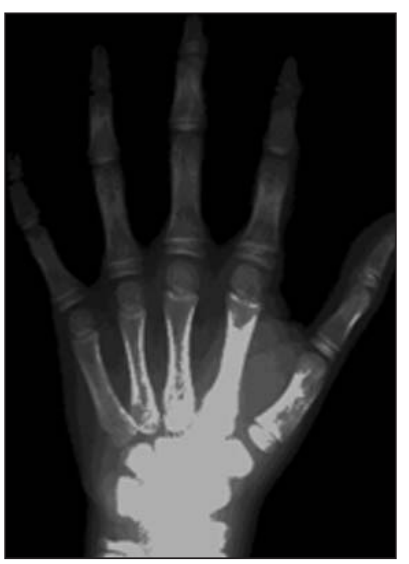

Fig 7:Child's Wrist and Hand, showing the clear lines at the end (epiphysis) of the long bones. These areas, which are made of cartilage, are the epiphyseal plates, where growth occurs.

\section{Visual treatment object (V.T.O):}

VTO helps in determining the patients ultimate goal and also to motivate the patient about the success of the treatment by activator which result aesthetic improvement. VTO helps in determinig mandibular manipulation to relocate the jaw in 
the direction of the treatment objectives. For example, a patient with a sk. class II malocclusion could be instructed to bring into a normal sagittal relation frequently thereby instantly creating an illusion of having correction of the malocclusion. This clinical procedure helps to realize the improvement. Class III (sk.) malocclusion could be similarly trained for improvement. VTO helps in motivation of the patient by visualizing change. This motivate the patient to use any appliance which is not otherwise aesthetically acceptable. It can be done-

- by using mirror

- by drawing picture

- by using cephalogram

- by comparing before and after treatment photographs or video of the treated patient or before and after wax bite registration of the patient to be treated

\section{Working Bite registration 8}
a. Class II activator
b. Class III activator

One important aspect of proper actvation fabrication is the determination and reproduction of the correct registration of desired "construction or working" bite.

The purpose of this mandibular manipulation is to relocate the jaw in the direction of the treatment objectives.

a. anterior positioning for class II malocclusion

b. Posterior positioning for class III malocclusion

This creates reorientation of orofacial muscles and generate functional forces . In this stage a careful attention must be given to study of the plaster model, cephalometric and functional pattern.

\section{Class II case :}

The usual intermaxillary relationship for average class II problem is edge-to-edge incisal. However, advancement of mandible should not exceed $8 \mathrm{~mm}$ (less advancement is advisable) or three quarters of the mesiodistal dimension of the 1st molar. At this stage any deviation of the mandible or mid-line shifting is also corrected

Opening of the bite: The vertical opening of the mandible should be $2-3 \mathrm{~mm}$ more than the free way space. (Vertical opening varies from $1 \mathrm{~mm}$ to $4 \mathrm{~mm}$ between incisor, depending on the curve of spee, the amount of forward positioning of mandible and direction of growth). In more advancement, vertical opening should minimum and vice versa. More vertical opening $(7 \mathrm{~mm}$ in molar region) is easily tolerable in permanent dentition. In mixed dentition it should be less. If forward 7 to $8 \mathrm{~mm}$, the vertical opening should 2 to $4 \mathrm{~mm}$ and if forward 3 to $5 \mathrm{~mm}$, the vertical opening should 4 to $6 \mathrm{~mm}$.

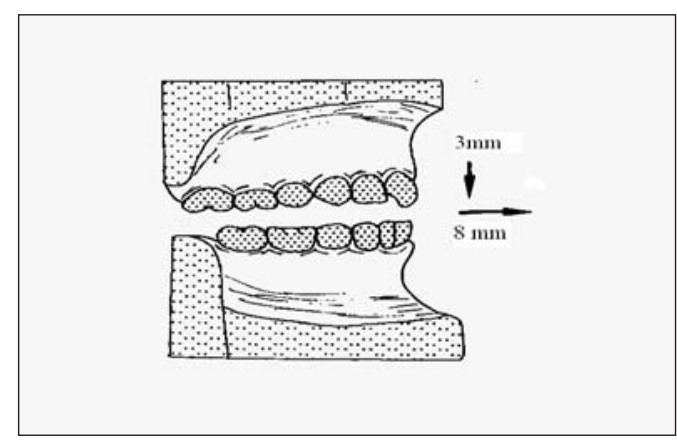

Fig.9: Construction bite in edge to edge relationship with slight vertical opening. Courttsey: Thomas Rakosi, In: Graber TM, Neumann Bedrich. Removable Orthodontic Appliance.

- If the antero-posterior discrepancy is two big anterior positioning of the mandible can be done in two or more stages.

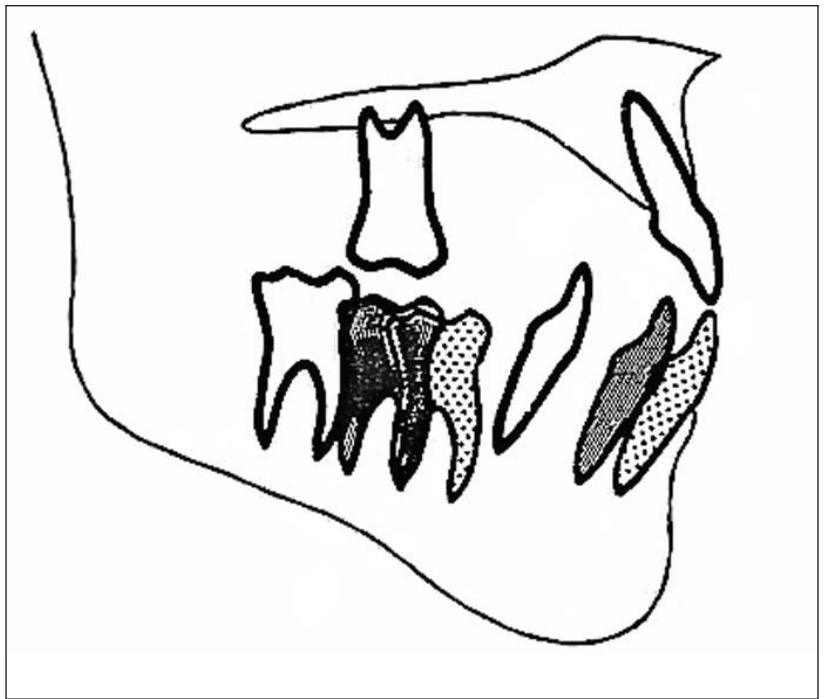

Fig.10: Anterior positioning of the mandible in two phase- First phase, dark, and second phase, dotted. Courttsey: Thomas Rakosi, In: Graber TM, Neumann Bedrich. Removable Orthodontic Appliance.

- In Class II div 1 malocclusion due to underdevelopment, the mandible should advanced to normal occlusion.

- In Class II div 1 malocclusion that are the result of mandibular underdevelopment and maxillary prognathism, maxillary bicuspids are removed and the anterior teeth moved back to extraction space as completely or half distance adjunct with mandibular advancement. 


\section{Class II div-2 case:}

\section{Options:}

1. At first prefunctional fixed attachment treatment is done to align the incisors then conventional class II activator is used.

2. If fixed attachment pretreatment is not used, the construction bite must be done insteps, shifting only to the most lingually inclined tooth and moving the lingually inclined incisors labially with springs in the first activator, then taking a new construction bite in a more forward position, which is possible. To save time while making a new appliance, the activator may be cut into two pieces at the occlusal plane and the lower half may be slide forward the desired position and reattached with self cure acrylic.

\section{Class III : (Illustrated case reports : figure 14-25)}

- Construction bite is taken by the retruding the lower jaw as much as possible and vertical opening best $1.5 \mathrm{~mm}$ to $3 \mathrm{~mm}$ between the incisal margin to allow the correction of anterior cross bite. If retruding less the vertical opening should more.

- In these cases it is possible to achieve an edge to-edge bite relationship with the posterior teeth out of contact

At this stage any deviation of the mandible or mid-line shifting is also corrected

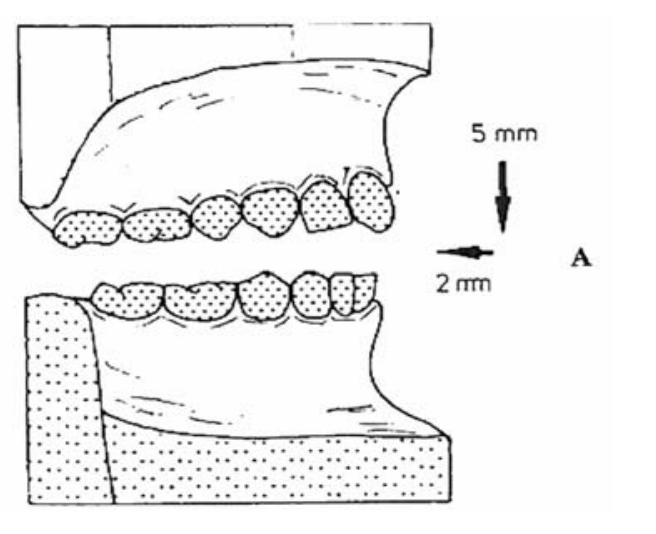

Fig. 11: The lower jaw is manipulated as far posteriorly as it will go or manual guided for construction bite. Courttsey: Thomas Rakosi, In: Graber TM, Neumann Bedrich. Removable Orthodontic Appliance.

\section{Open bite case:}

The dysfunction in open bite problems can be primary or secondary. Depending on the localization of dysfunction, the open bite can be anterior or posterior. In primary dysfunctions, in which abnormal muscle function is a major etiological factor, the growth pattern is usually average or horizontal. In open bite problems with a vertical growth pattern, the dysfunction is mostly secondary or adaptive.
Activator is not indicated for the treatment of skeletal open bite. Functional appliances are more likely to be successful in case within primary dysfunction and at least an average growth pattern. If the skeletal relationship is orthognathic the anterior positioning of mandible is not necessary, if there is disto occlusion, moving mandidle slightly forward and mandible should moving backward in class III tendency, vertical opening should be 4 to $5 \mathrm{~mm}$.

Purpose of bite registration

- To bring the lower jaw into a tolerable forward position.

- To ' block the bite'

- Depressing the lower anterior teeth.

- Stoping their eruption

- Stimulate eruption of posterior teeth.

\section{Procedure for bite Registration:}

\section{For Class II}

For construction bite maximum forward movement of mandible is $9 \mathrm{~mm}$ to $10 \mathrm{~mm}$ and optimal forward movement of mandible is usually half of the maximum range. More than half forward movement causes more uncomfortable for the patient and patient becomes less cooperative.The best positions for obtaining the desired histological transformation of the TMJ from class II malocclusion to Class I occlusion is approximately half of the distance that the can move forward along the anterior wall of the fossa of the articular tubercle. Greater than half along the articular eminence might prevent any favorable anatomical rebuilding of the TMJ structures.

\section{Method :}

Materials Required: a. Modeling wax 1 sheet, b. Warm water c. Upper and lower model, the lingual side in the first and or second permanent molar area should deep, which permit construction of a deep lingual flange and assists in holding the appliance in position. d. wax knife, e. pencil

\section{STEPS in bite registration:}

Step 1: In front of mirror, the patient is instructed to move the mandible into desired position. It is best obtained if the operator can guide the mandible.

Step 2: Reproduce the maximum forward movement of the mandible and correct occlusal clearance. Register the true mandibular midline with a pencil on the labial surface of the upper and lower incisors on the casts and in the patient's mouth.

Step 3: Determine the amount of mesial and vertical mandibular displacement of the construction bite. Mesial shift can be marked with a pencil on the buccal surface of the first molars on the cast. 
Step 4: Advice the patient to practice the desired movement of mandible. If needed, gently guide the mandible. Advice to move the mandible slowly to the desired position and to stop movement immediately when asked to do so.

Step 5: Soften a sheet of modeling wax with warm water and make a tight roll, approximately $1 \mathrm{~cm}$ in diameter.

Step 6: Shape the roll to conform to the lower dental cast.

Step 7: Press the soften roll on the lower cast so that the buccal teeth are covered but anteriorly the roll lies just lingual to lower incisors. Make a vertical groove on the wax to indicate midline.

Step 8: Remove any excess wax that extends on the retromolar area. The distal half of the last molar should not be covered with wax.

Step 9: Again soften it with Warm water.

Step 10: Transfer the wax roll to the patient's mouth, fitting it on the lower arch in the same manner that it was fitted on the lower cast.

Step 11: Move the mandible forward and advice to bite gently guide the mandible to correct midline, and asked to stop while desire vertical opening is achieved. If register fails, make a new roll and repeat the procedure.

Step 12: Remove the wax bite from the mouth and chill it on cold water. With sharp knife, trim the excess buccal wax until the occlusal margins of the molars are visible. By carefully checking the plaster casts, also remove all wax that is contracting the soft tissue, the inter-proximal area, and the palate. If this is not done, the wax bite cannot be seated properly on the casts.

Step 13: Place the wax bite between the casts and check that the mandible is moved in desired direction. If construction bite is incorrect, replace it on the lower cast, soften the superior surface on flame, and add a layer of warm wax. Repeat the procedure from Step 10 through Step 14.

Step 14: Replace the hard wax bite in the patient's mouth to assure the correct fit.

\section{Class III Construction bite (Fig. 11,14,15)}

The construction bite for class III case is taken in the most retruded or hinge-axis position of the mandible, with the vertical opening best $1.5 \mathrm{~mm}$ to $3 \mathrm{~mm}$ between the incisal margins to allow the correction of anterior cross bite.

\section{Method}

Instrument and material required: a. Modeling Wax 1 sheet, b. Warm water c. Upper and lower model, the lingual side in the first and or second permanent molar area should deep, which permit construction of a deep lingual flange and assists in holding the appliance in position. d. cold water. e. wax knife, f. pencil

\section{STEPS:}

Step 1: In front of mirror, the patient is instructed to move the mandible into desired position. It is best done if the operator can guide the mandible.

Step 2: Reproduce the most retruded or hinge-axis position of the mandible and correct occlusal clearance. Register the true mandibular midline with a pencil on the labial surface of the upper and lower incisors on the casts and in the patient's mouth.

Step 3: Advice the patient to practice the desired movement of mandible. If needed, gently guide the mandible. Advice to move the mandible slowly according to verbal instructions and to stop movement immediately when asked to do so.

Step 4: Soften a sheet of modeling wax with warm water and make a roll, approximately $1 \mathrm{~cm}$ in diameter.

Step 5: Shape the roll to conform to the lower dental cast.

Step 6: Press the soften roll on the lower cast so that the buccal teeth are covered but anteriorly the roll lies just lingual to lower incisors. Make a vertical groove on the wax to indicate midline.

Step 7: Remove any excess wax that extends on the retromolar area. The distal half of the last molar should not be covered with wax.

Step 8: Again soften it with slightly Warm water.

Step 9: Transfer the wax roll to the patient's mouth, fitting it on the lower arch in the same manner that it was fitted on the lower cast.

Step 10: Move the mandible backward and advice to bite gently guide the mandible to correct midline, and asked to stop while desired vertical opening achieve. If register fails, make a new roll and repeat the procedure.

Step 11: Remove the wax bite from the mouth and chill it. With sharp knife, trim the excess buccal wax until the occlusal margins of the molars are visible. By carefully checking the plaster casts, also remove all wax that is contracting the soft tissue, the inter-proximal area, and the palate. If this is not done, the wax bite cannot be seated properly on the casts. 
Step 12: Place the wax bite between the casts and check that the mandible is moved desired amount. If construction bite is incorrect, replace it on the lower cast, soften the superior surface on flame, and add a layer of warm wax. Repeat the procedure from Step 9 through Step 13.

Step 13: Replace the hard wax bite in the patient's mouth and have patient close the jaw slightly more firmly to assure the correct fit.

\section{Mechanism 10 \\ Class II: \\ a. Growth vectors that moves the lower jaw in forward direc- tion \\ b. The articular effectiveness of the activator moves the condyle in forward-downward position \\ c. The new position through condylar growth \\ d. Adaptation to the new position by remodeling in the fossa.}

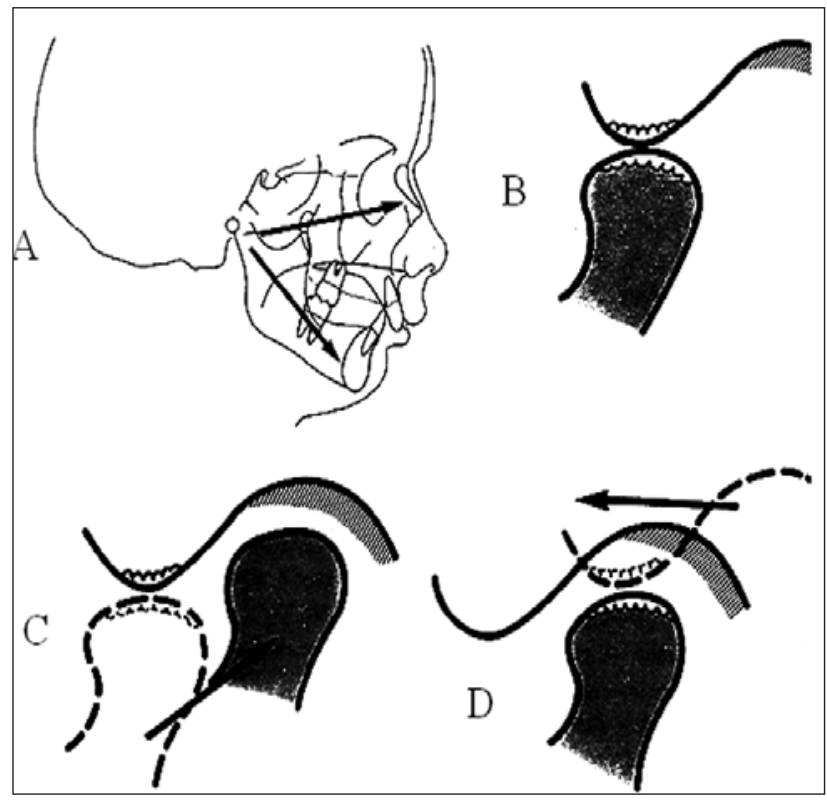

Fig. 12: A Growth vectors that moves the jaw forward direction (a) The articular effectiveness of the activator moves the condyle in forwarddownward position (b)The new position through condylar growth (c) Adaptation to the new position by remodeling in the fossa.(d)

Courtesy: TM Graver

\section{For Class III:}

It is in complete opposition to the kinetic concept of the class II activator.

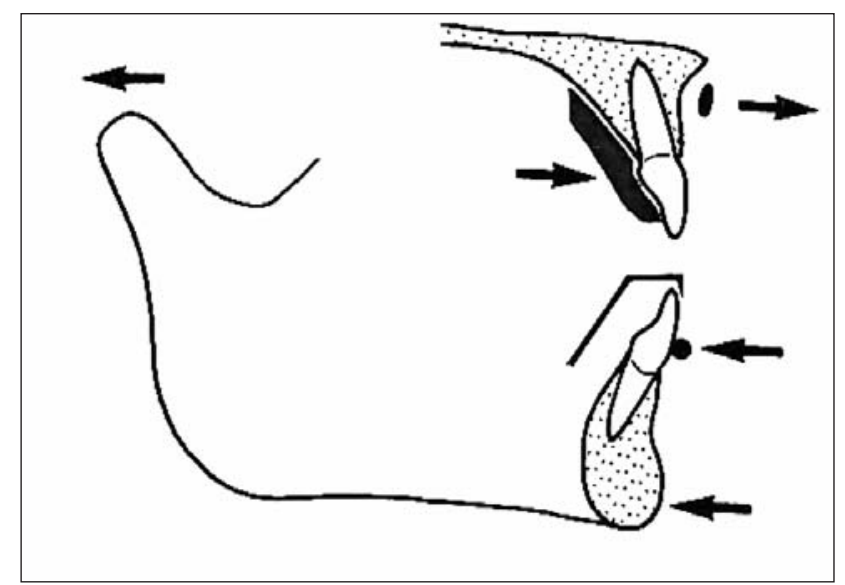

Fig. 13: A Growth vectors that moves the jaw backward direction Courttsey: Thomas Rakosi, In: Graber TM, Neumann Bedrich. Removable Orthodontic Appliance.

\section{Open bite:}

Controll tongue thrust and finger sucking.

Prevention of eruption of posterior teeth and eruption of anterior teeth is encouraged.

\section{Wire frame work:}

Spring, labial bow etc.

Fabricate a labial bow of $.8 \mathrm{~mm}$ or $.9 \mathrm{~mm}$ s.s wire with 'U' loops at the canine area.

- Placed two layer of relief tape ( $1 \mathrm{~cm}$ wide $)$ on the soft tissue area of the model over which the "U" loop is shaped.

- A length of wire is adapted to the labial surfaces of the incisors, at the junction of the gingival and middle third of the crowns. Any loop over the canine area should be have $1 \mathrm{~mm}$ to $1.5 \mathrm{~mm}$ clearance to the labial to permit freedom for erupting canine, the tags are design at the middle of the occlusal clearance.

\section{For class II: A maxillary labial bow is used.}

For class III: A mandibular labial bow is used to guide the mandible distally. In addition to a mandibular labial bow a maxillary labial bow is used. The maxillary labial bow is kept a slight distance way from the labial surfaces to relieve any lip pressure. ${ }^{4}$

5. Recheck the registered bite on the patient's mouth. The time spent on cheking the construction bite save the clinician the trouble of repeating the whole procedure of making an activator. Replace the hard wax bite in the patient's mouth and 
have patient close the jaw slightly more firmly to assure the correct fit. Check the amount of antero-posterior, vertical and lateral displacement precisely.

\section{Articulation of the registered bite (Fig. 16,17) :}

The easiest way of articulating the models is to use a standard, plane-line articulator and fix the casts with the incisor teeth facing towards the hinge of the articulator. ${ }^{11}$ So as to facilitate the manupulatiuon of acrylic resin or wax from the lingual aspect, while fabricating the activator.

Steps 1: Wax bite assembled on the casts. Then the casts are soaked in water at room temperature for five minutes.

Steps 2: Adjust the articulator so that the final coat of the plaster on the upper articulator arm should never be more than 5 to $6 \mathrm{~mm}$ thick

Step 3: A thick mix of plaster is placed on a clean ceramic tile.

Step 4: Seat the articulator (lower arm) on the mix of the plaster

Step 5: Place the casts over the plaster so that the incisor teeth face toward the hinge of the articulator or sideways.

Step 6: Place the mix of plaster over the upper model and close the articulator.

Step 7: Another layer of plaster is placed over the upper arm of the articulator.

Step 8: Finish the plaster with proper locked condition of the articulator.

\section{Fabrication of the appliance}

The activator can be fabricated by using auto polymerizing acrylic resin or by heat curing acrylic resin.

Fabrication of activator with self curing acrylic resin (Fig. 18 A, B, Fig. 19,20,21):

Step 1: The casts and articulator are soaked in water at room temperature for five minutes and the excess water is shaken off.

Step 2: two thin coat of separating medium are brushed on the casts, allowing the first coat to dry before applying the second coat.

Step 3: Place the wire components

Step 4: Fabricate upper and lower acrylic plate separately

Step 5: Close the articulator.

Step 6: A wax shell is formed around the buccal and labial surfaces of both arches by shaping a strip of modeling wax over the mounted casts.
Step 7: From the lingual side of the casts, liquid and powder are applied in sprinkle method to join the upper and lower plate as a single unit.

Step 8: Now the acrylic packed articulator is preserved for curing.

Step 9: After confirmation of curing, the wax sheet that covered the labial surfaces of the casts is removed and the labial and buccal extensions of the acrylic are inspected for any large air bubbles. Such spaces can be repaired with new addition of powder and liquid.

Step 10: The appliance is carefully seperated from the casts with a plaster knife that is placed under the posterior border of the palate and under the lingual border of the mandibular portion.

\section{Fabrication of activator with heat curing acrylic resin:}

Step 1: A wax pattern of the appliance should be made incorporating the wire work at first.

Step 2: Then it is flasked (Half Flasking, Full Flasking)

- A thin mix of plaster is brushed into the the impression of the various tooth surfaces in the wax to ensure that air bubbles are not trapped during flasking.

- Invest the fitting surfaces of the waxed appliance is in one half of the flask to avoid distorting the appliance during packing and pressing.

- The second half is poured after applying separating mediumto the lower half.

Step 3: Dewaxed

- When the plaster is set, the flask is heated in water and the halves separated, the wax pattern washed out.

Step 4: Packed with heat curing acrylic resin.

- Apply separating medium, the flask packed with acrylic materials.

Step 5: Cured to form the activator and after processing and cooling, the appliance is deflasked, cleaned and dried.

\section{Trimming and polishing of the appliance}

Excess acrylic material around the lower and posterior edges, is removed and the appliance trimmed and polished. The acrylic appliance is placed on the models which are returned to the articulator and registration of the vertical dimension checked. If the teeth do not meet evenly and exactly in their impression in the appliance, anteriorly and posteriorly adjust it by proper trimming. 
9. Insertion of the appliance in the oral cavity:

The activator is inserted in the patient mouth. If the teeth do not meet evenly and exactly in their impression in the appliance, anteriorly and posteriorly adjust it by proper trimming. Figure 22, 23

\section{Instruction for the appliance:}

Clear instruction should be given to the patient in presence of the parent on the use and maintenance of the appliance. It is one of the most important aspects of ensuring success with the appliance that the patient and parent should be adequately counseled. Verbal advice should be reinforced with a list of DO's and DON'T's

- With the aid of a mirror the patient should be shown how to remove and insert the appliance.

- Appliance should be worn almost full time a day and night (at least 14 hours a day) and to remove while brushing and swimming or playing.

- A high standard of oral hygiene should be insisted to avoid the possibility of enamel decalcification and gingival inflammation.

- The patient should be instructed to clean the appliance by brushing it with soap and water during tooth brushing. Care should be taken while cleaning so as not to bend / distort any component.

- Diet should be that required for good general health but avoid eating hard and sticky food and sweets.

- Immediately contact the orthodontist if the appliance hurts, become loose or any part is broken.

- Patient should be instructed not to leave the appliance out of the mouth for a long period of time as it enhances chances of getting damage or distorted.

- Patient having appliance with screws should be given instruction on how to activate the screws and frequency of activation.

- Appliance should be kept in water and away from the pet animals when it is not used by the patient.

- Continue with your routine dental visits.

- Patient should be assured that the initial difficulty in eating and speaking will go away soon with time.

- Patient should also be assured that the initial discomfort can be eased with the mild analgesic.

- Patient should be aware that failure to follow these instructions will prolong his/her orthodontic treatment.
11. Selective trimming and adding of the resin as adjustment of the appliance:

Where bucaal or labial movement of tooth is required, add the acrylic on that area of the activator and where lingual movement of tooth is required, trim the acrylic on that area of the activator.

For Class II : Trim the acrylic lingual to the upper incisors, distally to the teeth in the upper buccal segments and add acrylic on Lower anterior region labial side of the appliance, mesially to the teeth in the buccal segments. ${ }^{12}$

For Class III: Add the acrylic lingual to the upper incisors, trim mesially to the teeth in the upper buccal segments and trim the acrylic lingual to the Lower anterior region (labial side of the appliance), distally to the teeth in the buccal segments.

Note : The improvement of Class III relation (fig. 24-25)

\section{Sequential treatment procedure ${ }^{13}$}

The patient is usually checked every 4 weeks to 8 weeks. At the return appointments, the fit of the appliance and the progress of the treatment are observed. If there are any inaccuracies, the appliance should be ground away and stabilized by cold cure acrylic as indicated. The appliance is adjusted so as not to interfere with eruption. The proper wearing of the appliance should be checked carefully to observe the character of the mucosa on the posterior periphery of the appliance. As the treatment progress and the anteroposterior jaw relationship improves, the original activator occationally seems to become ineffective. Most of the time, it is best to make a new activator. However, if the activator still fits accurately in the mouth, the same activator can be adjusted with new construction bite.

\section{Indirect Method}

- Impression for new plaster casts and a new construction bite are first taken.

- Place the plaster casts together with the construction bite in articulator.

- Cut the activator horizontally along the free way space between the jaws.

- Put the separated halves of the appliance on the casts.

- Put a layer of cold cure acrylic on the cut and roughened side of the activator.

- Put the casts with the halves of the activator in place into the articulator and bring them together.

- After the acrylic has set, remove the surplus and polish the appliance again.

\section{Direct Method}

- Grind all interfering acrylic projections in the lower arch portion of the appliance so that the patient is able to move his mandible antero-posteriorly and keep the desired new construction bite position while the activator is firmly seated on the maxillary arch.

- The lower portion of the appliance may then be lined with soft wax. 
- Now the appliance is replaced in the mouth, and a new construction bite is taken.

- After the wax has been chilled, it cut of posterior to the premolar, and this area is thoroughly cleansed and roughened.

- When the jaws close, only the wax in the anterior portion is maintained in the new bite position.

- The uncovered areas are then filled with cold curing acrylic.

- The lingual mucosa itself is covered with a thin layer of petroleum and the appliance is put in the mouth to allow the acrylic to harden.

- The appliance is removed when the material is nearly set and the surplus ground off and polished.

- The remaining wax in the anterior portion is removed, the area cleansed and roughened, and the procedures is repeated to complete the new construction bite.

\section{Febrication of activetor and Illustrated case report}

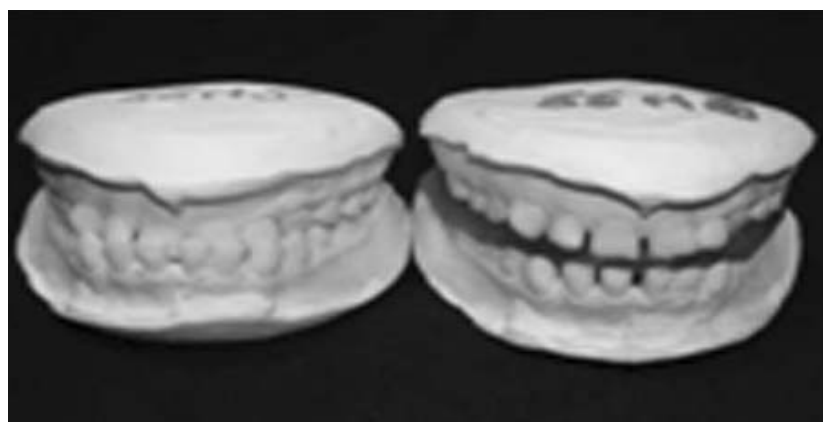

Fig. 14 A. Model in occlusion 14 B. Model with waxbite
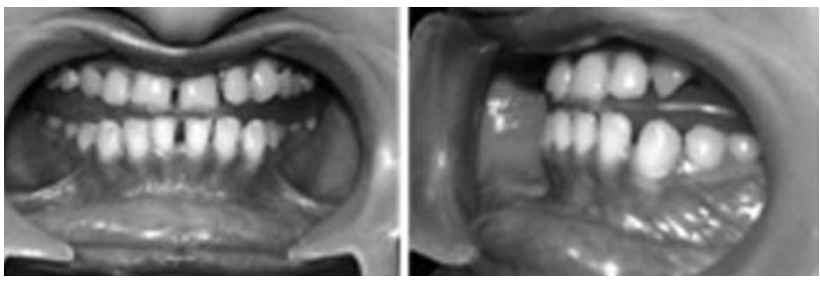

Fig. 15 AB. Intra oral photographs, Wax bite in mouth

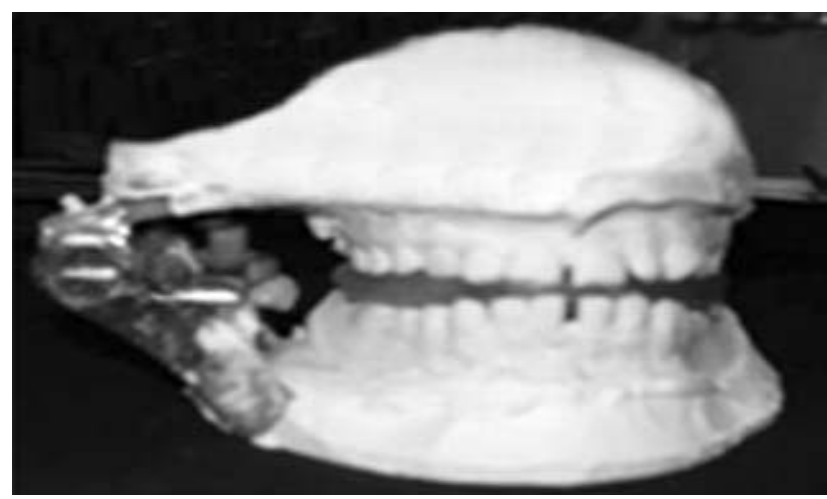

Fig. 16. Articulation of model with Wax bite

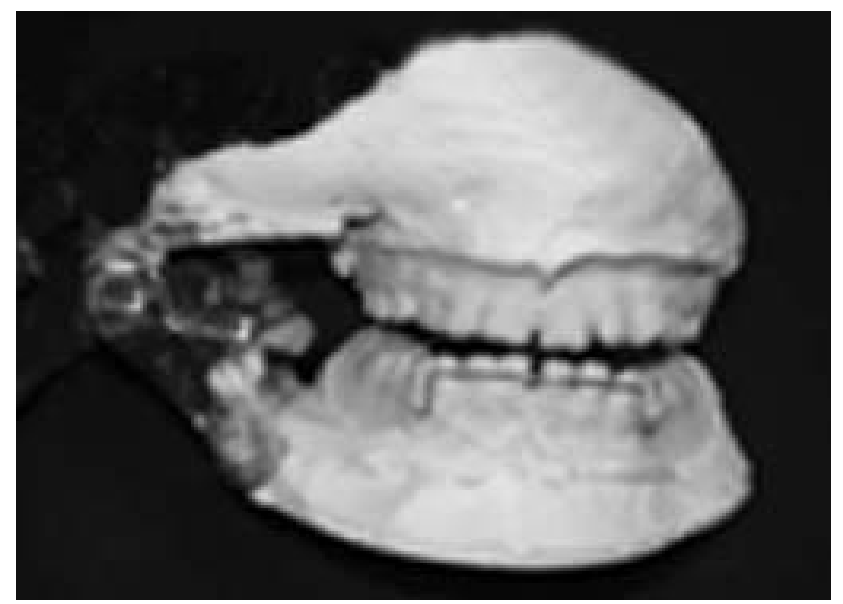

Fig. 17. Articulation of Model and lower labial insert in to the lower model

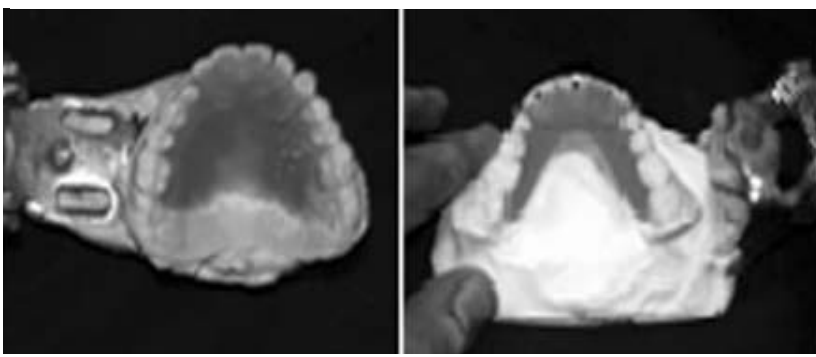

Fig. 18 A, B. Fabrication of upper and lower acrylic plate seperately

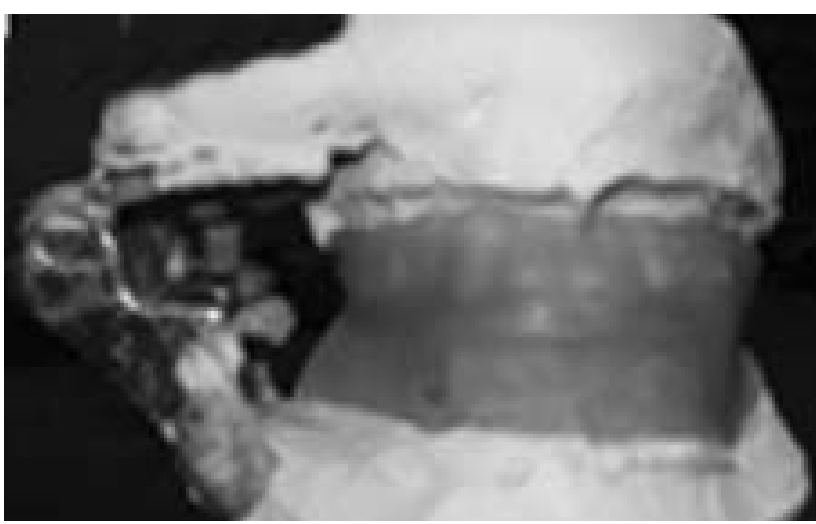

Fig. 19 A wax shell is formed around the buccal and labial surfaces of both arches by shaping a strip of modeling over the mounted cast
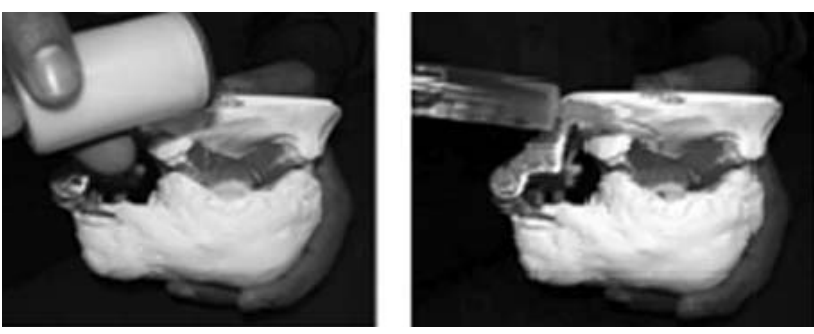

Fig. 20. From the lingual side of the casts, liquid and powder are applied in sprinkle method to join the upper and lower plate as a single unit. 


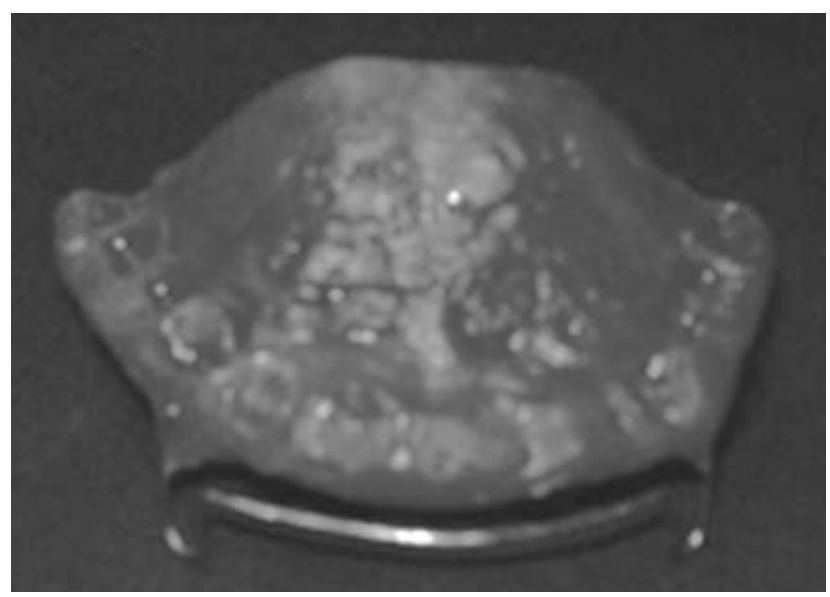

Fig. 21. Class III activator
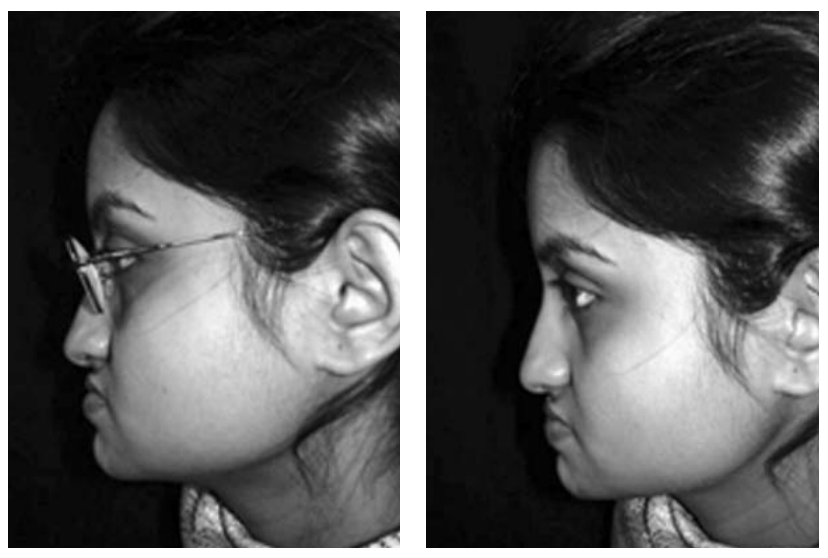

Fig. 22A. Facial photograph lateral view (before treatment) 22B. Facial photograph lateral view (activator within the mouth)

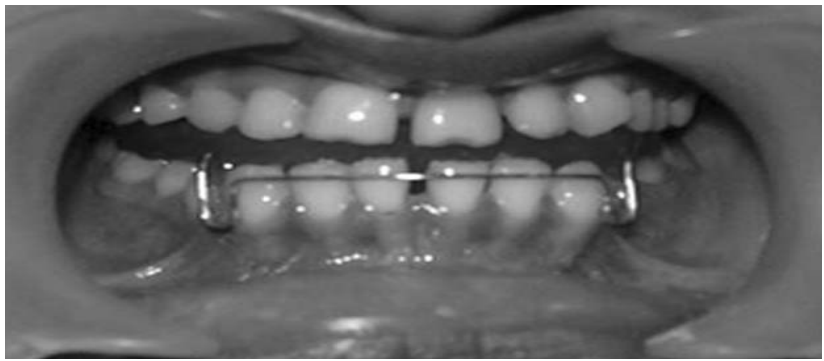

Fig. 23. Intra oral photograph (activator within the mouth)
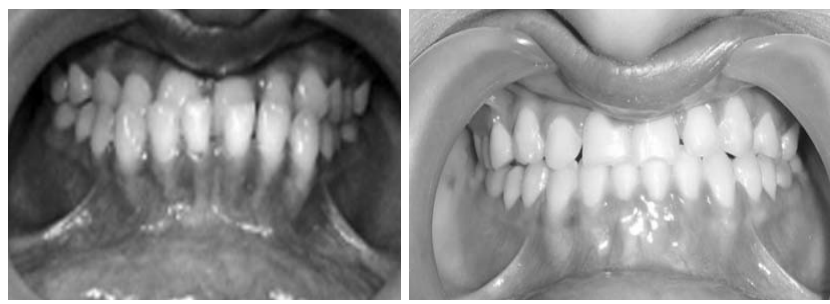

Fig. 24. Intra oral photograph A. Before treatment and B. After treatment
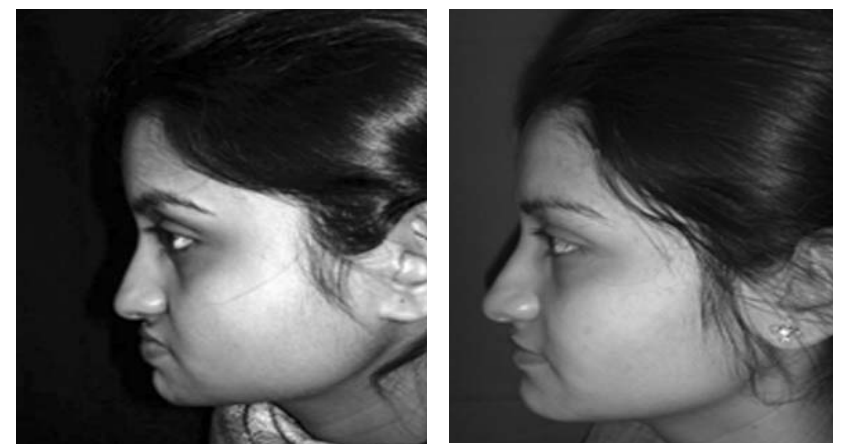

Fig. 25. Facial photograph A. Before treatment and B. After treatment, better face profile

\section{Reference:}

1. Robert E Moyers. Orthodontic technique. In: Handbook of Orthodontics. 4th Ed.1988; pp 511- 560.

2. S I Bhalajhi. Myofunctional Appliance. In: Orthodontics The Art and Science. 3rd Ed. Reprint.2006; pp 329-364

3. Tulley WJ, Houston W J B. Functinal Appliance . In: A Textbook of Orthodontics 1989; 244-257

4. Graber TM, Neumann Bedrich. The activator: Use and modification. In: Removable Orthodontic Appliance 2nd ed. 1984; 198-239

5. Hossain MZ, Tarique A A M, Haque S, Khaleque KA. Two stage treatment of a pseudoskeletal Class III. J of Oral Health Oct' 2003;5(2), 24-27

6. Masataka Hisano, Kunihiko Ohtsubo, Chooryung J. Chung, Fajar Nastion, and Kunimichi Soma (2006) Vertical Control by Combining a Monoblock Appliance in Adult Class III Overclosure Treatment. The Angle Orthodontist: March 2006, Vol. 76, No. 2, pp. 226-235.

7. Kharbanda Om P. Class III malocclusion in growing patients. In: Orthodontics Diagnosis and management of Malocclusion and Dentofacial deformities. First ed. Elsevier 2009. pp124-151.

8. Graber TM, Neumann Bedrich. Construction bite. In: Removable Orthodontic Appliance 2nd ed. 1984; pp175-197

9. Rakosi T. Cephalometric diagnosis for a functional appliance therapy. In: Removable Orthodontic Appliance 2nd ed. 1984; pp102-127

10. Rakosi T. Principles of Functional Appliance In: Graber TM, Rakosi T, Petrvic AG. Dentofacial Orthopedics with Myofunctional Appliance. St Louis .CV Mosby, 1997; 85-106

11. C. Philip Adams, W. John . S. Kerr. Functional Appliance. In: The Design , Constructions and Use of Removable Orthodontic Appliance. Sixth ed. 1995; 98-150

12. Kinast, G. R. In Schwarz, A. M. : Lehrgang der Gebissregelung, 2nd ced. , Vol. II. Vienna, Urban and Schwarzenberg, 1956

13. Donald G woodside. The Harvold- Woodside activator In: Graber TM, Neumann Bedrich. Removable Orthodontic Appliance 2nd ed. 1984; 244-309

\section{Correspondence:}

Prof. Dr. Md. Zakir Hossain, BDS, PhD

Prof. and Head,

Dept. of Orthodontics and Dentofacial Orthopaedics,

Dhaka Dental College and Hospital, Mirpur-14, Dhaka 1206.

E-mail:hossainzortho@yahoo.com,

web:www.orthodentalcare.com 